\title{
Depression is Associated With Sexual Risk Among Men who Have Sex With Men, but is Mediated by Cognitive Escape and Self-Efficacy
}

\author{
Lisa M. Alvy, ${ }^{1,2}$ David J. McKirnan, ${ }^{1,2}$ Gordon Mansergh, ${ }^{3}$ Beryl Koblin, ${ }^{4}$ Grant D. Colfax, ${ }^{5}$ \\ Stephen Flores, ${ }^{3}$ Sharon Hudson, ${ }^{6}$ and the Project MIX Study Group \\ ${ }^{1}$ Psychology Department, University of Illinois at Chicago, Chicago, Illinois \\ ${ }^{2}$ Howard Brown Health Center, Chicago, Illinois \\ ${ }^{3} \mathrm{CDC}$, Division of HIV/AIDS Prevention, Atlanta, Georgia \\ ${ }^{4}$ New York Blood Center, New York, New York \\ ${ }^{5}$ San Francisco Department of Public Health, San Francisco, California \\ ${ }^{6}$ Health Research Association, Los Angeles, California
}

Send proofs to:

Lisa M. Alvy

1007 W. Harrison St., M/C 285

Psychology Department

University at Illinois at Chicago

Chicago, IL 60607

Phone: (312) 996-2540 ext. 2589

Fax: (312) 413-4122

Ealvy2@uic.edu

Note: The findings and conclusions in this report are those of the authors and do not necessarily represent the views of the CDC. 


\begin{abstract}
Men who have sex with men (MSM) show high rates of HIV infection, and higher rates of depression than non-MSM. We examined the association between depression and sexual risk among "high risk" MSM. Evidence has been mixed regarding the link between depression and risky sex, although researchers have rarely considered the role of psychosocial vulnerabilities such as self-efficacy for sexual safety or "escape” coping styles. In a national sample $(N=1,540)$ of HIV-positive and HIV-negative MSM who reported unprotected sex and drug use with sex partners, we found evidence that depression is related to HIV transmission risk. Self-efficacy for sexual safety and cognitive escape mediated the link between depression and risk behavior, suggesting that psychosocial vulnerability plays an important role in the association of depression with sexual risk. These findings may help us construct more accurate theories regarding depression and sexual behavior, and may inform the design of sexual safety interventions.
\end{abstract}

Key words: depression; sexual risk; MSM; cognitive escape; self-efficacy 


\section{INTRODUCTION}

MSM constitute $64 \%$ of the adult and adolescent men with HIV, ${ }^{1}$ and despite widespread knowledge of modes of HIV transmission, HIV incidence rates remain high in this group. ${ }^{1}$ At the same time, converging empirical evidence points to higher rates of depression in men who have sex with men (MSM) compared to non-MSM. ${ }^{2}$ In an extensive mental health study conducted in the Netherlands, the 12-month and lifetime prevalence of major depression and dysthymia (i.e., less severe, chronic depression) was nearly three times as high in MSM as in non-MSM, ${ }^{3}$ and national household and population-based studies in the United States demonstrate similar findings. ${ }^{4-6}$ Given the high rates of depression in this community, we examined the link that depression may have with HIV infection among MSM.

The health behavior literature suggests that those with depression are less likely to tend to their physical health generally. In those with diabetes, major depression is associated with lower adherence to diabetes medication, less physical activity, and poorer nutrition, all of which contribute to worse health outcomes. ${ }^{7,8}$ More broadly, university students who report depressive symptoms are more likely to engage in everyday unhealthy behaviors including a lack of physical activity, not eating breakfast, and keeping irregular sleep hours. ${ }^{9}$

There are reasons to suspect that depression and related negative affect will lead to risky health behaviors as well. In both the coping and self-regulation literature there is a consistent finding that those who experience negative affect are more likely to engage in avoidant coping or affect-based regulation, as opposed to instrumental problem-solving. ${ }^{10-12}$ Avoidant coping has also been found to be associated with unprotected sex. ${ }^{13,14}$ In one sample of gay HIV-positive men, avoidant coping was the best psychosocial predictor of unprotected anal intercourse (UAI) with an HIV-negative or unknown status partner. ${ }^{13}$ 
From a cognitive escape perspective, individuals may cope with negative mood states by attempting to escape cognitive awareness of behavioral norms, narrowing their attention to more immediate, pleasurable outcomes. ${ }^{15,16}$ The concept of cognitive escape is similar to theories involving alcohol or drug expectancies, ${ }^{17}$ but frames substance use as a specific form of avoidant coping. Studying a high-risk MSM sample, McKirnan and colleagues ${ }^{18}$ found that men who combined drugs with sex to cognitively escape awareness of HIV risk were more likely to engage in sexually risky activities. Although researchers have not explored a relationship between depression and cognitive escape, conceptual similarities between escape and avoidant coping suggest that escape tendencies may be higher in depressed MSM.

An additional pathway whereby depression may lead to risky sexual behavior is selfefficacy for sexual safety. Self-efficacy for sexual safety - i.e., one's confidence in his or her ability to practice safer sex consistently - has been widely studied in the health behavior literature. ${ }^{19}$ Depression and depressed mood are known predictors of low self-efficacy. ${ }^{20}$ In the smoking cessation literature, depression has been shown to contribute to low self-efficacy to refrain from smoking, ${ }^{21}$ a health behavior that requires cognitive effort, self-control, and is repetitive, like condom use. Because self-efficacy for effortful health behaviors is likely to be lower in those with depression, ${ }^{21}$ low self-efficacy for sexual safety may contribute to decreased condom use among depressed MSM.

Qualitative research with MSM in community settings suggests that depression and negative affect are strongly associated with risky sex. During group intervention sessions of a recent MSM sexual behavior intervention, feelings of sadness and loneliness emerged as consistent themes related to sexual risk. ${ }^{22}$ Similarly, interview data collected by Bancroft and 
colleagues $^{23}$ revealed that some gay men described engaging in unsafe sex when depressed because they were less concerned about the consequences.

Despite qualitative evidence, quantitative research has been less consistent in demonstrating a link between depression and riskier sex. ${ }^{24-27}$ For example, using data from the Urban Men's Health Study, Stall and colleagues ${ }^{24}$ did not find an association between high scores on the Center for Epidemiological Studies Depression (CES-D) scale (scores above 23) and UAI with a serodiscordant or unknown HIV status partner. However, in the EXPLORE study Koblin and colleagues ${ }^{26}$ uncovered a longitudinal effect of depression on unprotected sex when breaking depressed MSM into severity categories. The research team found that men with moderate depressive symptoms were significantly more likely to have contracted HIV than those who were not depressed or were severely depressed.

Inconsistency in the depression and sexual risk literature may be due to differences in study populations or depression measurement. An additional possibility is that the effect of depression on sexual risk is complex, such that it is mediated by other psychosocial vulnerabilities. For example, Strathdee and colleagues ${ }^{28}$ found that although "sexual risk-takers" had higher depression scores on the CES-D, this relationship was no longer significant when they took factors such as low social support, nitrate use, less education, and history of sexual abuse into account. Some of these variables might have mediated a depression-risk relationship, although the authors did not report such an analysis. Beck and colleagues ${ }^{29}$ demonstrated a link between depression and sexual risk that was mediated by erroneous sexual risk cognitions. They found that the relationship between depression and riskier sex among MSM disappeared when they controlled for cognitions such as believing that fidelity or only practicing insertive sex offers sufficient protection against STIs. 
Prior studies on depression and sexual behavior have underemphasized the importance of psychosocial vulnerabilities. Using self-report data from a national HIV prevention study, we explored the relationship between depression and risk for transmitting HIV among sexually risky, substance-using MSM. Given the literature on depression, self-care, and risk, we predicted that depression would be associated with HIV transmission risk, and that cognitive escape, avoidant coping, and self-efficacy for sexual safety would partially mediate this relationship.

\section{METHODS}

The data used in these analyses are from baseline assessments collected as part of Project MIX, a four-city behavioral intervention for sexual safety, delivered to high-risk MSM who reported a recent history of drug or alcohol use with sex. Project staff collected baseline data from November 2004 through July 2006. Men volunteered for a cohort study with a 3-, 6-, and 12-month follow-up during which they received six, 2-hour group intervention sessions (either experimental or attention control). Collaborating research sites were the New York Blood Center and the New York Academy of Medicine, Howard Brown Health Center and the University of Illinois at Chicago, the San Francisco Department of Public Health AIDS Office, and the Health Research Association in Los Angeles. The Centers for Disease Control and Prevention (CDC) served as a Cooperative Agreement funding source and a research partner on this project.

\section{Participants}

Participants represented a convenience sample of MSM who reported at least two instances of alcohol use or one instance of non-injection drug use during or immediately prior to sex, and reported UAI with an unknown HIV status or an HIV serodiscordant male partner, all within the prior six months. Eligible participants agreed to participate in a six-session, groupbased intervention, and complete interviews at baseline and at 3-, 6-, and 12-month follow-ups. 
Individuals were ineligible if they admitted to illicit injection drug use other than methamphetamine (e.g., heroin) and prescribed medications, had a plan to move from their respective city in the next 15 months, or were currently involved in another study trial.

Project staff recruited participants using both active and passive methods. Active recruitment included outreach into street and venue locations where MSM socialized or congregated, such as bars, clubs, gay businesses, and agencies; and through websites or extant email databases or listservs at each site. Men were passively recruited through flyers and information cards, project websites and ads, and referrals from local agencies and organizations, community advisory board members, and other study participants. The research team designed the active and passive recruitment approaches to elicit a statistical representation of African American, Latino, and Caucasian participants, and near equal numbers of HIV-negative and HIV-positive men.

\section{Procedure}

Project staff conducted a brief telephone screen with all potential participants to determine eligibility. Staff directly called men whose names were collected during active recruitment, and fielded calls from men who responded to passive recruitment. Project staff scheduled an initial visit with those who screened eligible, which consisted of informed consent, a confirmatory eligibility screening, the baseline assessment, and HIV testing and counseling. Men who knew themselves to be HIV-positive could bring evidence of their serostatus (e.g., HIV medications) in lieu of testing. We collected the baseline data via audio computer-assisted selfinterviews (ACASI) prior to providing HIV test results. Participants completed the ACASI in a private, confidential setting, with a project assistant available to answer questions or clarify the 
use of the computer. Institutional Review Boards at the Centers for Disease Control and Prevention and each local institution approved all study procedures.

\section{Measures}

ACASI topics included sexual behavior, psychosocial covariates including mood and sex-related cognitions, alcohol and drug use, and demographic information (for a full description

see Mansergh et al. ${ }^{30}$ ). All items were from standard instruments in the field and used simple check boxes or rating scales with appropriate skip patterns. Median completion time was 45 minutes. This analysis focused on a subset of questions related to sexual behavior and psychosocial variables, described below.

\section{Descriptive Characteristics}

Descriptive characteristics included standard indicators of ethnicity, age, education, annual income, and self-reported HIV status. Respondents reported their date of birth to obtain an exact age, but reported all other characteristics categorically, including income, for which respondents selected one of nine income categories based on their range of income.

\section{Sexual Activity and HIV Transmission Risk}

We assessed sexual behavior with an extensive ACASI section addressing the three months prior to the interview. To index overall sexual activity we counted the number of partners participants reported any anal sex with during the previous three months. To assess $H I V$ transmission risk partners we counted the total number of sex partners with whom the participant reported any UAI whose serostatus was unknown or discordant from the participant's status (i.e., transmission risk partners). This included both primary and non-primary partners, and both insertive and receptive UAI. Both measures were highly positively skewed. To correct skewness we truncated each index at its $99^{\text {th }}$ percentile. 


\section{Psychosocial Measures}

Psychosocial measures represented a range of sexual attitudes or risk dispositions, using both standard scales and scales developed for this study. Constructs and scale composition used in these analyses are listed below.

Depression. We assessed depression with seven items from the Center for Epidemiological Studies Depression scale (CES-D). ${ }^{31}$ We selected these items from Santor and Coyne's 9-item short version of the scale, ${ }^{32}$ dropping two positively worded items because of low item-total correlations. Participants rated items such as "[How often have you] felt sad [during the past week]?" on frequency scales ranging from "never or rarely" to "mostly or always (5-7 days)," $\alpha$ $=.88$. For descriptive purposes, we assessed depression with a symptom count, consistent with Santor and Coyne's procedure. ${ }^{32}$ We counted any item endorsed with "often (3-4 days)" or "mostly or always (5-7 days)" as a symptom. For all other analyses, we created a mean severity score (0-4) from all seven depression items to capture all the within sample variance.

Self-efficacy for sexual safety. We measured self-efficacy for sexual safety with seven facevalid items assessing participants' confidence in practicing safer sex. Participants rated the following items on a five-point scale ranging from "strongly disagree" to "strongly agree": "I can choose safer sex with a man I have sex with regularly;" "I am able to avoid behavior that may put me at risk of HIV infection;" "When I am drunk or high, I can avoid situations that I consider sexually risky;" "If I ever did something risky, I am confident that I would go back to having safer sex right away;" "I am confident that I can have safer sex even if my partner really doesn't want to;" "I never lose site of what I consider safer sex, no matter what I am feeling or if I am drunk or high;" and "When I am drunk or high, I can choose safer sex with a man I have never had sex with before." The 7-item alpha was .72. We identified MSM as especially low in self- 
efficacy when they reported that they "strongly disagree" or "somewhat disagree" (versus "strongly agree," "somewhat agree," or "neither") that they could be sexually safe in more than half of the scenarios described (i.e., four or more scenarios).

Avoidant coping. We assessed avoidant coping by taking the mean of the 4-item Behavioral Disengagement subscale of the COPE, ${ }^{33}$ reflecting avoidant coping in response to stress, plus a fifth item, "I've been giving up the attempt to cope," written for this study to increase the reliability of this subscale. The 5-item alpha was .76. Participants rated items on a four-point scale ranging from "I usually don't do this at all" to "I usually do this a lot." For descriptive purposes, we identified MSM who endorsed over half of these items (i.e., three items or more) with a '2,' '3,' or '4', as engaging in avoidant coping regularly (i.e., high in avoidant coping).

Cognitive escape. We measured cognitive escape with a 7-item scale we previously developed to assess the use of sex and drugs for cognitive escape purposes. Participants rated the following items on a five-point scale ranging from "strongly disagree" to "strongly agree": "When I am high or drunk I am more likely to have sex with people I ordinarily wouldn't have sex with;" "When I am high or drunk I am more likely to want to have anal sex with a man;" "Being drunk makes me more comfortable sexually;" "After getting drunk or high I am more sexually responsive;" "When I am drunk or high I find it difficult to stay within my sexual limits;" "Once I have the chance to have sex, I can't say no even if we don't use condoms;" and "When I am drunk or high I will do anything with anyone". The 7-item alpha was .71. For descriptive purposes, we categorized MSM as frequently engaging in cognitive escape (i.e., high in cognitive escape) when they agreed (i.e., selected '4') or strongly agreed (i.e., selected '5') with more than half of the escape items (versus indicating that they "strongly disagree," “somewhat disagree," or "neither"). 


\section{Data Analysis}

To describe this cohort of men in terms of depression severity, we grouped the complete baseline sample of MSM into three categories based on their number of depression symptoms. We categorized participants who endorsed four or more symptoms on the CES-D as high in depression, those reporting one to three symptoms as moderate in depression, and those who did not endorse any depression symptoms as low in depression. Our high depression criterion is consistent with Santor and Coyne's 4-item cut-off indicating clinical depression, ${ }^{32}$ although ours is a more restrictive criterion given that our scale had two fewer items. Additionally, to identify the degree of psychosocial vulnerability in this group of MSM, we calculated the percentage of participants who were high in avoidant coping and cognitive escape, and low in self-efficacy for sexual safety using the criteria described above.

For all analyses we included age, income, education level, ethnicity, HIV status, and research site as covariates. To explore the relationship between depression and transmission risk, we tested the effects of depression on risk using linear regression methods. We entered both depression and transmission risk as continuous variables.

To examine the extent to which self-efficacy for sexual safety, avoidant coping, and cognitive escape mediated the association of depression and transmission risk, we used the classic Baron and Kenny ${ }^{34}$ approach to mediation but applied a significance testing procedure recommended by MacKinnon, Fairchild, and Fritz. ${ }^{35}$ The MacKinnon approach determines the significance of the mediational (i.e., indirect) path by estimating confidence intervals based on the distribution of the product of the 'a' (independent variable to mediator) and ' $b$ ' (mediator to dependent variable, controlling for the independent variable) regression paths. This approach to mediation testing provides a more accurate assessment of statistical significance than approaches 
that compare the ' $a b$ ' product to a normal distribution. ${ }^{36}$ After testing the significance of the ' $a$ ' and ' $b$ ' paths for each set of variables, we used the PRODCLIN program designed by MacKinnon and colleagues ${ }^{37}$ to compute the critical values for the distribution of the product. If the $95 \%$ confidence intervals does not include zero, the mediation effect is statistically significant at $p<.05$.

To obtain a measure of effect size we used the standardized betas of the direct and indirect effect of depression on risk from each analysis to compute the proportion mediated. Given that 1,540 MSM were in the study sample, this commonly used approach to effect size estimation is considered stable and appropriate. ${ }^{35}$

\section{RESULTS}

\section{Descriptive, Psychological, and Behavioral Characteristics of the Sample}

At baseline, 1,540 MSM participated in the ACASI assessment, approximately evenly distributed across the four study sites (see Table I). The sample had a near equal representation of HIV-positive and HIV-negative MSM, and had strong representation of African American, Caucasian, and Latino men. Given the relatively small percentage of Asian Americans and Pacific Islanders, and the difficulty in interpreting the "mixed" and "other" ethnicity categories, we combined these three groups for covariate purposes. The majority (68\%) of the sample made less than $\$ 30,000$ a year. Participants had a normal distribution across education categories (for additional information on sample demographics see Mansergh et al. ${ }^{30}$ ).

Categorizing participants by depression severity yielded 611 participants (40\%) low in depression, 594 participants (39\%) moderate in depression, and $335(22 \%)$ participants high in depression. Psychosocial vulnerability, as indicated by measures of avoidant coping, cognitive escape, and low self-efficacy for sexual safety, was high in this sample of MSM. Half of the 
sample (51\%), reported regular engagement in avoidant coping strategies by endorsing three or more avoidant coping items out of five, and $61 \%$ of the sample reported frequent engagement in cognitive escape by endorsing more than half of the escape items. A quarter of the sample (25\%) reported especially low self-efficacy, indicating low efficacy in four or more scenarios.

This sample of MSM reported a high level of sexual activity. Over half (56\%) of the men had five or more sexual partners in the past three months, and $10 \%$ reported more than 20 partners. Participants also reported high levels of transmission risk. Seventy-two percent of the sample $(n=1,117)$, had at least one transmission risk partner in the past three months, defined as having a UAI partner of serodiscordant or unknown HIV status. The average number of transmission risk partners was close to three $(M=3, S D=5)$.

\section{Depression and Transmission Risk}

Entering depression as a continuous variable into a standard regression equation revealed a significant, although modest, linear association of depression and transmission risk (i.e., UAI) partners, $F(1,1523)=10.45, p<.01, R^{2}=.007$. As depression increased, so did risk.

\section{Mediation Analyses}

The three psychosocial variables we tested as mediators were modestly correlated (selfefficacy and avoidant coping, $r=-.10$; avoidant coping and cognitive escape, $r=.18$; cognitive escape and self-efficacy, $r=-.22$; all $p \mathrm{~s}<.05)$. Given that the relationships were not strong and the variables represent different constructs, we conducted identical but separate analyses for each.

All three variables were related to depression. As expected, higher levels of depression predicted higher levels of avoidant coping, $F(1,1523)=396.42, p<.001, R^{2}=.188$, and greater use of cognitive escape strategies, $F(1,1523)=152.96, p<.001, R^{2}=.089$. Higher levels of 
depression also predicted lower levels of self-efficacy for sexual safety, $F(1,1523)=32.12, p<$ $.001, R^{2}=.020$.

To assess the next component of mediation, we tested whether these variables predicted the outcome of interest, transmission risk, when controlling for depression. Self-efficacy for sexual safety was a significant predictor of risk, controlling for depression, $F(1,1522)=41.71, p$ $<.001, R^{2}=.026$, as was cognitive escape, $F(1,1522)=6.69, p<.05, R^{2}=.004$. Avoidant coping did not predict transmission risk with depression in the model, $F<1$, indicating that this variable did not mediate the depression-risk relationship.

We used MacKinnon and colleagues' statistical program to test for the significance of the overall mediation effect for self-efficacy and cognitive escape. ${ }^{37}$ The $95 \%$ confidence interval for the product of the coefficients was 0.09 to 0.25 for self-efficacy, and 0.04 to 0.27 for cognitive escape. Because neither interval includes zero, these results demonstrate that self-efficacy and cognitive escape are mediators of the effect of depression on transmission risk partners.

Comparing direct and indirect effects of depression on transmission risk, we found that self-efficacy and cognitive escape served as partial mediators (see Figure 1). When we controlled for self-efficacy for sexual safety, the prediction of risk from depression was still significant, $F(2,1522)=26.22, p<.001, R^{2}=.032$, but the standardized beta for depression decreased from .084 in the indirect model to .060 in the direct model, representing $29 \%$ mediation. Similarly, when we controlled for cognitive escape, the model predicting transmission risk was also significant, $F(2,1522)=8.59, p<.001, R^{2}=.011$. The standardized beta for depression decreased from .084 to .062 , representing $26 \%$ mediation.

Given that self-efficacy for sexual safety and cognitive escape individually served as partial mediators of the depression and risk relationship, we sought to test the combined 
mediating effect of these variables. Because MacKinnon's PRODCLIN program does not allow for multiple mediators, ${ }^{37}$ we used a composite variable to explore the extent to which selfefficacy for sexual safety and cognitive escape jointly mediate the association of depression with transmission risk. We created this composite vulnerability variable by combining the seven items from the self-efficacy and cognitive escape scales, $\alpha=.78$.

As expected, higher levels of depression predicted higher levels of combined vulnerability, $F(1,1523)=138.40, p<.001, R^{2}=.080$. Vulnerability was also a significant predictor of risk, controlling for depression, $F(1,1522)=34.77, p<.001, R^{2}=.021$, thus satisfying another component of mediation. When entered into the PRODCLIN program, we found that vulnerability was a significant mediator of the depression and transmission risk association (CI: 0.20 to 0.44 ). The standardized beta for depression decreased from .084 to .038, representing 55\% mediation (see Figure 1). When we controlled for vulnerability, the model predicting transmission risk from depression was no longer statistically significant, indicating that the combined vulnerability measure served as a full mediator.

\section{DISCUSSION}

Using baseline data from a national HIV behavioral intervention trial, we explored the association of depression and risk for HIV transmission in HIV-negative and HIV-positive MSM. Past studies have produced inconsistent findings on MSM depression and sexual risk, ${ }^{27}$ and researchers have offered several explanations for these discrepancies. ${ }^{26,27,38}$ In this study, we investigated the possibility that the relationship between depression and sexual risk is complex, such that psychosocial vulnerabilities mediate the link between depression and risk.

Consistent with theory and qualitative research, we found that participants with more symptoms of depression reported a greater amount of transmission risk. To explore the effect of 
depression on risk, we tested whether the association would be mediated by three key psychosocial vulnerabilities: avoidant coping, cognitive escape, and self-efficacy for sexual safety. Self-efficacy and cognitive escape were partial mediators of the depression to risk link, each accounting for more than a quarter of the variance in the association of depression and risk. Combined, these variables mediated the depression-risk association, mediating over half of the relationship. This suggests that self-efficacy and cognitive escape were important mediators of the depression and risk association in this cohort. Given that the combined mediation effect of self-efficacy and cognitive escape was equivalent to the sum of their separate effects, these two variables appear to be distinct mediators of the association of depression and risk.

Contrary to prediction, avoidant coping did not serve as a mediator. Given that avoidant coping was highly correlated with depression it is not surprising that the association of avoidant coping with transmission risk was not significant with depression in the model. Also, cognitive escape may be a better measure of avoidant-like coping when exploring sexual risk. Cognitive escape specifically captures the use of sex and substances for escape purposes, whereas avoidant coping reflects a more general response to stress.

One potential limitation of our analytical approach is the way we combined the escape and efficacy indices to create an overall measure of psychosocial vulnerability. Conceptually these variables are linked: low self-efficacy for instrumental coping is hypothesized to make escape coping more likely. ${ }^{16}$ However, they are not viewed as equivalent constructs. Our combining them here was supported in part by the relatively strong alpha of the joint scale and our desire to test the shared variance of the two measures in a single analysis.

Because the data were cross-sectional we were unable to do a strong test of mediation. It is possible that the casual relationships between these variables are reversed, such that low self- 
efficacy or cognitive escape lead to depression, or that engaging in risky sexual activity contributes to depression and/or psychosocial vulnerability. Although these causal directions are plausible, they may not be primary. Converging lines of research support the casual relations asserted here: depression can erode one's self-efficacy and lead to cognitive escape, both of which contribute to risky sexual behavior.

There are several possible reasons why depression and sexual risk demonstrated an association in this dataset but have not in others. One explanation is the characteristics of this sample. Participants in Project MIX were especially risky, ethnically diverse, and engaged in a high level of substance use compared to other large-scale samples of MSM (e.g., the Urban Men's Health Study ${ }^{24}$; the EXPLORE Study ${ }^{26}$ ). Because of these drug-use and sexual patterns, participants in MIX who were depressed may have been more likely to engage in escape coping processes, and thus sexual risk, than MSM in other samples.

An escape model would predict that men who have low self-efficacy for instrumental coping and who have socially sanctioned "escape" mechanisms available to them - such as sex and drugs - would have a low threshold for responding to depression via escape behaviors. ${ }^{16}$ Consistent with that, both low-self efficacy and an escape coping index mediated the effect of depression on risk in Project MIX. Since we selected a sample known to be high in sexual risk and drug use - which could be characterized as "externalizing" responses to depression - an escape model may well characterize their risk behavior. This may differ for less explicitly risky samples of MSM, for whom depression may manifest in more "internalizing" responses such as withdrawal. Mustanski offered a similar symptom profile explanation for inconsistent findings in the depression and risk literature. ${ }^{38}$ 
Given that the MIX cohort had very high levels of sexual risk and substance use they may represent "core group transmitters" of HIV and STIs among MSM. ${ }^{39,40}$ As such, conceptual models of their risk behavior are particularly important to developing targeted preventive interventions. Screening for depressive symptomatology could enhance prevention efforts. ${ }^{41}$ Many health care centers already screen for depression using instruments such as the PHQ-9, and clinically significant scores on such measures could flag a need for services. Standard Cognitive Behavior Therapy (CBT) and anti-depressant medication could be used in this endeavor. ${ }^{42}$

Our findings here suggest that cognitive escape strategies and low self-efficacy may be especially important to target in future HIV prevention initiatives among high risk MSM. Although our qualitative data suggested that depression was a precursor to sexual risk for many men, these results indicate that the effect of depression on risk must be understood and potentially modified by addressing these more proximal psychosocial variables. Having men attend to their coping strategies as a preventive intervention may be a fruitful adjunct to addressing general issues such as depression or specific issues such as condom use methods. Coping skills training may also be more amenable to both public health campaigns and paraprofessional interventions.

Because of the modest association of depression and transmission risk in this study and the unique characteristics of our sample, researchers should continue to investigate the depression and risk relationship among MSM. The MIX cohort is important from a public health perspective, but does not allow us to generalize these findings to all MSM. Additionally, given that our sampling methods may have excluded more severely depressed men, future investigations could focus on this group to explore whether depression has different implications for risk at higher levels of severity. To make depression severity comparisons across samples, it 
would be helpful for researchers to use standardized depression instruments, such as the full CES-D, when possible.

All analyses reported here are between-subjects and cross-sectional and thus do not explore the role of depression or mood states within an individual, or over time. Negative affect experienced throughout the course of a day or week may have different effects than multiple and enduring symptoms of depression. Researchers have begun to examine state affect as it relates to sexual risk in MSM, ${ }^{38}$ and additional research is indicated. ${ }^{43}$ Longitudinal analyses could help to elucidate the casual effects of depression and psychosocial vulnerability on HIV risk behavior.

These findings suggest that escape coping processes and mental health, generally, are important to understanding sexual behavior among high risk MSM. Given the ongoing spread of HIV, prevention efforts targeting depression and psychosocial vulnerability should be developed and tested. 


\section{ACKNOWLEDGEMENTS}

This research was conducted as part of Project MIX: Behavioral Intervention to Reduce Sexual Risk Behavior of Substance-Using (Non-Injection) Men Who Have Sex With Men. It was supported by the Centers for Disease Control and Prevention through cooperative agreements with the University of Illinois at Chicago and Howard Brown Health Center, New York Blood Center and the New York Academy of Medicine, the San Francisco Department of Public Health AIDS Office, the Health Research Association in Los Angeles, and the CDC Division of HIV/AIDS Prevention in Atlanta. The authors wish to recognize the contributions of the Project MIX Study Team. We would also like to thank Lisa Kuhns, Steve DuBois, Christine Holland, David Fingerhut, and Natalie Richie for their comments on this paper, as well as Brian Mustanski, Linda Skitka, and Jon Kassel for their help on an earlier version of this paper. 


\section{REFERENCES}

1) CDC. Cases of HIV infection and AIDS in the United States and dependent areas, 2006. In: U.S. Department of Health and Human Services C, ed. Vol 18: U.S. Department of Health and Human Services, CDC, 2008.

2) Herek GM, Garnets LD. Sexual orientation and mental health. Annu Rev Clin Psychol. 2007;3:353-75.

3) Sandfort TG, de Graaf R, Bijl RV, Schnabel P. Same-sex sexual behavior and psychiatric disorders: findings from the Netherlands Mental Health Survey and Incidence Study (NEMESIS). Arch Gen Psychiatry. 2001 Jan;58(1):85-91.

4) Mills TC, Paul J, Stall R, Pollack L, Canchola J, Chang YJ, et al. Distress and depression in men who have sex with men: the Urban Men's Health Study. Am J Psychiatry. 2004 Feb;161(2):278-85.

5) Cochran SD, Mays VM, Sullivan JG. Prevalence of mental disorders, psychological distress, and mental health services use among lesbian, gay, and bisexual adults in the United States. J Consult Clin Psychol. 2003 Feb;71(1):53-61.

6) Cochran SD, Mays VM. Lifetime prevalence of suicide symptoms and affective disorders among men reporting same-sex sexual partners: results from NHANES III. Am J Public Health. 2000 Apr;90(4):573-8.

7) Lin EH, Katon W, Von Korff M, Rutter C, Simon GE, Oliver M, et al. Relationship of depression and diabetes self-care, medication adherence, and preventive care. Diabetes Care. 2004 Sep;27(9):2154-60.

8) Odegard PS, Capoccia K. Medication taking and diabetes: a systematic review of the literature. Diabetes Educ. 2007 Nov-Dec;33(6):1014-29; discussion 30-1.

9) Allgower A, Wardle J, Steptoe A. Depressive symptoms, social support, and personal health behaviors in young men and women. Health Psychol. 2001 May;20(3):223-7.

10) Folkman S, Lazarus RS. Stress-processes and depressive symptomatology. J Abnorm Psychol. 1986 May;95(2):107-13.

11) Dombeck MJ, Siegle GJ, Ingram RE. Cognitive interference and coping strategies in vulnerability to negative affect. Hillsdale, NJ, England: Lawrence Erlbaum Associates, Inc; 1996.

12) Holahan CJ, Moos RH, Holahan CK, Brennan PL. Social context, coping strategies, and depressive symptoms: an expanded model with cardiac patients. J Pers Soc Psychol. 1997 Apr;72(4):918-28. 
13) Semple SJ, Patterson TL, Grant I. Psychosocial predictors of unprotected anal intercourse in a sample of HIV positive gay men who volunteer for a sexual risk reduction intervention. AIDS Educ Prev. 2000 Oct;12(5):416-30.

14) Folkman S, Chesney MA, Pollack L, Phillips C. Stress, coping, and high-risk sexual behavior. Health Psychol. 1992;11(4):218-22.

15) Heatherton TF, Baumeister RF. Binge eating as escape from self-awareness. Psychol Bull. 1991 Jul;110(1):86-108.

16) McKirnan DJ, Ostrow DG, Hope B. Sex, drugs and escape: a psychological model of HIVrisk sexual behaviours. AIDS Care. 1996 Dec;8(6):655-69.

17) Cooper ML, Frone MR, Russell M, Mudar P. Drinking to Regulate Positive and Negative Emotions - a Motivational Model of Alcohol-Use. Journal of Personality and Social Psychology. 1995;69(5):990-1005.

18) McKirnan DJ, Vanable PA, Ostrow DG, Hope B. Expectancies of sexual "escape" and sexual risk among drug and alcohol-involved gay and bisexual men. J Subst Abuse. 2001;13(12):137-54.

19) Wulfert E, Wan CK. Condom use: a self-efficacy model. Health Psychol. 1993 Sep;12(5):346-53.

20) Maciejewski PK, Prigerson HG, Mazure CM. Self-efficacy as a mediator between stressful life events and depressive symptoms. Differences based on history of prior depression. $\mathrm{Br} \mathrm{J}$ Psychiatry. 2000 Apr;176:373-8.

21) John U, Meyer C, Rumpf HJ, Hapke U. Self-efficacy to refrain from smoking predicted by major depression and nicotine dependence. Addict Behav. 2004 Jul;29(5):857-66.

22) Veluz A, McKirnan DJ, Alvy LM. Qualitative analysis of HIV risk and motivation to change in drug-using men who have sex with men (MSM). American Public Health Association Conference. Washington, DC; 2007, November.

23) Bancroft J, Janssen E, Strong D, Vukadinovic Z. The relation between mood and sexuality in gay men. Arch Sex Behav. 2003 Jun;32(3):231-42.

24) Stall R, Mills TC, Williamson J, Hart T, Greenwood G, Paul J, et al. Association of cooccurring psychosocial health problems and increased vulnerability to HIV/AIDS among urban men who have sex with men. Am J Public Health. 2003 Jun;93(6):939-42.

25) Perdue T, Hagan H, Thiede H, Valleroy L. Depression and HIV risk behavior among Seattlearea injection drug users and young men who have sex with men. AIDS Educ Prev. 2003 Feb;15(1):81-92. 
26) Koblin BA, Husnik MJ, Colfax G, Huang Y, Madison M, Mayer K, et al. Risk factors for HIV infection among men who have sex with men. Aids. 2006 Mar 21;20(5):731-9.

27) Crepaz N, Marks G. Are negative affective states associated with HIV sexual risk behaviors? A meta-analytic review. Health Psychol. 2001 Jul;20(4):291-9.

28) Strathdee SA, Hogg RS, Martindale SL, Cornelisse PG, Craib KJ, Montaner JS, et al. Determinants of sexual risk-taking among young HIV-negative gay and bisexual men. J Acquir Immune Defic Syndr Hum Retrovirol. 1998 Sep 1;19(1):61-6.

29) Beck A, McNally I, Petrak J. Psychosocial predictors of HIV/STI risk behaviours in a sample of homosexual men. Sex Transm Infect. 2003 Apr;79(2):142-6.

30) Mansergh G, Flores S, Koblin B, Hudson S, McKirnan D, Colfax GN. Alcohol and drug use in the context of anal sex and other factors associated with sexually transmitted infections: results from a multi-city study of high-risk men who have sex with men in the USA. Sex Transm Infect. 2008 Nov;84(6):509-11.

31) Radloff LS. The CES-D Scale: A self-report depression scale for research in the general population. Applied Psychological Measurement. 1977 Sum;1(3):385-401.

32) Santor DA, Coyne JC. Shortening the CES-D to improve its ability to detect cases of depression. Psychological Assessment. 1997 Sep;9(3):233-43.

33) Carver CS, Scheier MF, Weintraub JK. Assessing coping strategies: a theoretically based approach. J Pers Soc Psychol. 1989 Feb;56(2):267-83.

34) Baron RM, Kenny DA. The moderator-mediator variable distinction in social psychological research: conceptual, strategic, and statistical considerations. J Pers Soc Psychol. 1986 Dec;51(6):1173-82.

35) MacKinnon DP, Fairchild AJ, Fritz MS. Mediation analysis. Annu Rev Psychol. 2007;58:593-614.

36) MacKinnon DP, Lockwood CM, Williams J. Confidence Limits for the Indirect Effect: Distribution of the Product and Resampling Methods. Multivariate Behavioral Research. 2004 Jan;39(1):99-128.

37) MacKinnon DP, Fritz MS, Williams J, Lockwood CM. Distribution of the product confidence limits for the indirect effect: program PRODCLIN. Behav Res Methods. 2007 Aug;39(3):384-9.

38) Mustanski B. The influence of state and trait affect on HIV risk behaviors: a daily diary study of MSM. Health Psychol. 2007 Sep;26(5):618-26. 
39) Boily MC, Lowndes C, Alary M. The impact of HIV epidemic phases on the effectiveness of core group interventions: insights from mathematical models. Sex Transm Infect. 2002 Apr;78 Suppl 1:i78-90.

40) Coxon APM, McManus TJ. How many account for how much? Concentration of high-risk sexual behaviour among gay men. Journal of Sex Research. 2000 Feb;37(1):1-7.

41) Chesney MA, Koblin BA, Barresi PJ, Husnik MJ, Celum CL, Colfax G, et al. An individually tailored intervention for HIV prevention: baseline data from the EXPLORE Study. Am J Public Health. 2003 Jun;93(6):933-8.

42) DeRubeis RJ, Hollon SD, Amsterdam JD, Shelton RC, Young PR, Salomon RM, et al. Cognitive therapy vs medications in the treatment of moderate to severe depression. Arch Gen Psychiatry. 2005 Apr;62(4):409-16.

43) Kalichman SC, Weinhardt L. Negative affect and sexual risk behavior: comment on Crepaz and Marks (2001). Health Psychol. 2001 Jul;20(4):300-1. 


\section{FIGURE CAPTION}

Figure 1. Association of depression and HIV transmission risk mediated by a) self-efficacy for sexual safety, b) cognitive escape; and, c) a joint self-efficacy (SE) and cognitive escape (CE) measure.

$\stackrel{* *}{p} \leq .001, \stackrel{*}{p}<.05$ 
Table I

Descriptive Characteristics of the Sample

Characteristic $n(\%)$

Age (in years)

$18-25$

$242(16 \%)$

$26-35$

$432(28 \%)$

$36-50$

$766(50 \%)$

51 or older

$96(6 \%)$

Study site

Chicago

Los Angeles

New York

San Francisco

HIV-Status

HIV-positive

HIV-negative

Unknown status

Ethnic identification

Caucasian

African American

Latino

Mixed ethnic background
$413(27 \%)$

$348(23 \%)$

$362(24 \%)$

$415(27 \%)$

$724(47 \%)$

$682(44 \%)$

$130(9 \%)$
$583(38 \%)$

$493(32 \%)$

$294(19 \%)$

$84(6 \%)$ 
Asian American or Pacific Islander 40 (3\%)

Other ethnic group $\quad 42(3 \%)$

Annual income

$\begin{array}{lc}\text { Less than } \$ 10,000 & 538(35 \%) \\ \$ 10,000-\$ 19,999 & 282(18 \%) \\ \$ 20,000-\$ 29,999 & 218(14 \%) \\ \$ 30,000-\$ 39,999 & 165(11 \%) \\ \$ 40,000-\$ 59,999 & 168(11 \%) \\ \$ 60,000 \text { or more } & 165(11 \%)\end{array}$

Education completed

Less than high school degree $\quad 108(7 \%)$

High school diploma or GED $\quad 376(25 \%)$

$\begin{array}{ll}\text { Some college } & 524(34 \%)\end{array}$

B.A. or B.S. degree $337(22 \%)$

Post-baccalaureate degree $191(12 \%)$ 